\title{
Anomalous turbulent velocity scaling from the Navier-Stokes equation
}

\author{
Jens Eggers ${ }^{\text {a,b }}$ and Siegfried Grossmann ${ }^{\text {a }}$ \\ a Fachbereich Physik, Philipps-Universität, Renthof 6, W-3550 Marburg, Germany \\ b Department of Mathematics, The University of Chicago, 5734 University Avenue, Chicago, IL 60637, USA
}

Received 8 March 1991; accepted for publication 8 April 1991

Communicated by B. Fricke

\begin{abstract}
For the first time corrections to classical $2 / 3$ scaling of the structure function of high Re turbulence are evaluated from the Navier-Stokes equation. The probability distribution for the velocity fluctuations develops increasingly stretched tails at smaller scales. The method employed to analyse the Navier-Stokes equation is a spatially resolved Fourier-Weierstrass decomposition. To make the equations numerically tractable we only simulate a small portion of real space, the other contributions are represented by renormalized coupling constants.
\end{abstract}

\section{Introduction}

Turbulent fluid flow $\boldsymbol{u}(\boldsymbol{x}, t)$ at very high Reynolds number $R e$ is characterized, among others, by the scaling exponents $\zeta(m)$ of the structure functions

$D^{m}(r)=\left\langle\left\langle|\boldsymbol{u}(\boldsymbol{x}+\boldsymbol{r}, t)-\boldsymbol{u}(\boldsymbol{x}, t)|^{m}\right\rangle \propto \propto \boldsymbol{r}^{\zeta(m)}\right.$.

《\ denotes the ensemble average, ideally translational invariant and isotropic. The classical exponents $\zeta_{\mathrm{cl}}(m)=m / 3$ follow from a simple dimensional argument, if one identifies the energy dissipation per unit mass $\epsilon$ as the only relevant physical parameter in the inertial subrange [1].

Experimentally, there are small corrections $[2,3]$ $\delta \zeta(m)$, due to intermittency, i.e. an increasing spatial spottiness of turbulent activity at smaller scales. So far there is no accepted theory of intermittency. While $\zeta_{\mathrm{cl}}(m)$ can be derived from mean field type analyses $[4,5]$ of the Navier-Stokes equation, intermittency corrections have been included by phenomenological models without dynamical justification [6-9]. A recently published numerical study of a dynamical cascade model [10] reveals intermittency, but the model only makes contact with the Navier-Stokes equation through energy conservation, which is known to be insufficient to fix the exponents [11]. In fact, in ref. [12] we demonstrate that the type of local interaction approximation employed in ref. [10] leads to classical scaling, if the Navier-Stokes interaction is correctly accounted for.

We recently introduced [13] a cascade model which is stated directly in terms of the velocity field. $\boldsymbol{u}$ is decomposed into modes localized both in $\boldsymbol{k}$ - and $\boldsymbol{x}$-space. Those types of decomposition have a long history [14], the first application to turbulence to our knowledge being due to Siggia [15]. Recent applications include the wavelet analysis of turbulent flow signals [16].

Study of high Re number turbulent flow by numerical analysis of the Navier-Stokes equation is possible only if the number of admitted modes is properly restricted. The basic idea in our previous paper [12] is to select a geometrically scaling set of momenta. The Fourier series then becomes of Weierstrass type. $\mathrm{Re} \approx 10^{6}$ could be dealt with. We now in addition introduce a corresponding geometrically refined spatial resolution. A pruning technique keeps the number of modes still tractable.

The space-momentum resolving mode hierarchy and the equations of motion resulting from the Navier-Stokes equation are presented in section 2. The main results (section 3 ) are: (i) The spatially coherent turbulent solutions considered in ref. [12] are unstable against spatially localized perturbations 
leading via Navier-Stokes interaction to enhanced, spotty fluctuations. (ii) The fluctuations amplify under repeated eddy decay. (iii) This leads to power law corrections $\delta \zeta(\mathrm{m})$ which agree with experiment. (iv) The probability of small as well as of large velocity deviations is enhanced, the Gaussian distribution becoming exponential-type with decreasing scale. This picture of intermittency is supported by a very recent wavelet analysis of a spectral simulation of turbulent Navier-Stokes flow [17].

\section{The self-similar mode system}

We start with the Fourier decomposition into plane waves $\exp (i p \cdot x)$ as usual. With the basic periodicity box chosen as $(2 \pi L)^{3}$ the momenta have the components $p_{i}=n_{i} L^{-1}$ with $n_{i}=0, \pm 1, \pm 2$, etc.

To deal appropriately with the many scales present in turbulent flow, we introduced [12] a hierarchical subset $\mathrm{K}$ of wavenumbers $\boldsymbol{p}$ partitioned into levels $\mathrm{K}_{l}, \mathbf{K}=\bigcup_{l} \mathrm{~K}_{l}$, each of which contains wavenumbers of approximately equal size. The smallest wavenumbers (or largest scales) defining level $l=0$ are $\mathrm{K}_{0}=\left\{\boldsymbol{p}^{(0)} \mid \boldsymbol{L p}^{(0)}= \pm(2,2,2), \pm(-1,2,2), \pm(2\right.$, $-1,-1), \pm(1,4,4), \pm(4,1,1),+$ all permutations $\}$. The larger wavenumbers (or smaller scales) are characterized by $\mathbf{K}_{l}=\left\{\boldsymbol{p}^{(l)} \mid \boldsymbol{p}^{(l)}=2^{l} \boldsymbol{p}^{(0)}, \boldsymbol{p}^{(0)} \in \mathrm{K}_{0}\right\}$. The wavenumber sets $K_{l}$ are geometrically scaled replica of $\mathrm{K}_{0}$. The smallest scale level $l=N_{\eta}$ defines the inner scale $\eta=\left(\nu^{3} / \epsilon\right)^{1 / 4}$ of the flow by $\eta \approx 1 /$ $p^{\left(N_{\eta}\right)}$. There are 13 wavevectors per level corresponding to 52 real mode amplitudes per level [12].

The Navier-Stokes equation for incompressible flow by $(\boldsymbol{u} \cdot \operatorname{grad}) \boldsymbol{u}$ and pressure gradient defines an interaction between the Fourier amplitudes $u_{i}(\boldsymbol{p}, t)$. It reads $-\mathrm{i} M_{i j k}(\boldsymbol{p}) \sum_{\boldsymbol{q}} u_{j}(\boldsymbol{q}, t) u_{k}(\boldsymbol{p}-\boldsymbol{q}, t)$, with $M_{i j k}(\boldsymbol{p})$ $=\left[p_{j} P_{i k}^{\perp}(\boldsymbol{p})+p_{k} P_{i j}^{\perp}(\boldsymbol{p})\right] / 2, P^{\perp}$ being the transverse projection. We now restrict the interaction to those momenta $\boldsymbol{p}, \boldsymbol{q}, \boldsymbol{q}^{\prime}=\boldsymbol{p}-\boldsymbol{q}$ which besides conserving momentum $p=q+q^{\prime}$ all belong to the representative hierarchical set $K$. This restriction automatically implies that the interaction is local in $p$-space: the vectors of an interacting triad either must lie in the same or in two neighbouring levels $\mathrm{K}_{l}$.

Next we want to include the physical fact that smaller scales should also be finer resolved in real space. We therefore divide the periodicity volume on the $l$ 's cascade level into boxes of linear extension $2 \pi L \times 2^{-1}$, each of which is represented by independent local Fourier amplitudes. Let $\mathbf{B}_{l}$ be the set of $l$ boxes, having $2^{3 l}$ elements. Denote the characteristic functions of the boxes as $\chi_{m}^{(l)}(t)(x)$, being 1 only if $2 \pi L \times 2^{-l} m_{i}^{(l)} \leqslant x_{i} \leqslant 2 \pi L \times 2^{-l}\left(m_{i}^{(l)}+1\right), i=1,2,3$, $m_{i}^{(l)}$ integer, $0 \leqslant m_{i}^{(l)} \leqslant 2^{l}-1$, and zero elsewhere. The velocity field $u_{i}(x, t)$ is now expanded into the space-momentum resolving Weierstrass-Fourier series

$$
\begin{aligned}
& u_{i}(\boldsymbol{x}, t) \\
& =\sum_{l=0}^{N_{\eta}} \sum_{\boldsymbol{p}(l) \in \mathrm{K} l} \sum_{, \boldsymbol{m}(l) \in \mathrm{B} l} u_{i}\left(\boldsymbol{p}^{(l)}, \boldsymbol{m}^{(l)}, t\right) \psi_{\boldsymbol{p}, \boldsymbol{m}}^{(l)}(\boldsymbol{x}) .
\end{aligned}
$$

The $u_{i}\left(\boldsymbol{p}^{(l)}, \boldsymbol{m}^{(l)}, t\right)$ are the complex mode amplitudes; the modes themselves are

$$
\begin{gathered}
\psi_{p, m}^{(l)}(x)=\left(2 \pi L \times 2^{-l}\right)^{-3 / 2} \\
\quad \times \exp \left(i p^{(l)} \cdot x\right) \chi_{m}^{(l)}(x)(x) .
\end{gathered}
$$

The modes are normalized but in general not mutually orthogonal. For any pair $p_{1}, p_{2}$ of the restricted wavenumber set $\mathrm{K}$, however, they are orthogonal (the proof using $2^{-l} L p_{i}^{(l)}$ integer).

Inserting the orthogonal space-momentum resolving Fourier-Weierstrass decomposition (2) into the Navier-Stokes equation leads to the coupled set of o.d.e.'s for the mode amplitudes:

$$
\begin{aligned}
& \frac{\partial \hat{u}_{i}\left(\boldsymbol{p}^{(l)}, \boldsymbol{m}^{(l)}, t\right)}{\partial t} \\
& =-\mathrm{i} M_{i j k}\left(\boldsymbol{p}^{(l)}\right)\left(\sum_{\boldsymbol{q}, \boldsymbol{p}-\boldsymbol{q} \in \mathbf{K},} \hat{u}_{j}\left(\boldsymbol{q}, \boldsymbol{m}^{(l)}\right) \hat{u}_{k}\left(\boldsymbol{p}-\boldsymbol{q}, \boldsymbol{m}^{(l)}\right)\right. \\
& +\sum_{q \in \mathrm{K}_{J}-1, \boldsymbol{p}-\boldsymbol{q} \in \mathrm{K} /} \hat{u}_{j}\left(\boldsymbol{q}, \boldsymbol{m}^{-}\left(\boldsymbol{m}^{(l)}\right)\right) \hat{u}_{k}\left(\boldsymbol{p}-\boldsymbol{q}, \boldsymbol{m}^{(l)}\right) \\
& \left.+\frac{1}{8} \sum_{\substack{q, p-q \in K^{\prime}\left(+1 \\
m^{+}\left(m^{(l)}\right)\right.}} \hat{u}_{j}\left(\boldsymbol{q}, \boldsymbol{m}^{+}\right) \hat{u}_{k}\left(\boldsymbol{p}-\boldsymbol{q}, \boldsymbol{m}^{+}\right)\right) \\
& -\nu \boldsymbol{p}^{(l) 2} \hat{u}_{i}\left(\boldsymbol{p}^{(l)}, \boldsymbol{m}^{(l)}\right)+\hat{f}_{i}\left(\boldsymbol{p}^{(l)}, \boldsymbol{m}^{(l)}\right) .
\end{aligned}
$$

These equations are very similar to the previous ones [12]. The only but crucial difference is that the $l$-level momenta $\boldsymbol{p}^{(l)}$ in an l-level spatial box $\boldsymbol{m}^{(l)}$ are now allowed to interact with the $(l+1)$-level momenta of all eight spatial subboxes $\boldsymbol{m}^{+}\left(\boldsymbol{m}^{(l)}\right)$ on $(l+1)$-level 
which branch off $m^{(l)}$. The first term is the interaction within the $l$-level, $\boldsymbol{m}^{(l)}$-box; the second one describes the interaction with the larger predecessor $\boldsymbol{m}^{-}\left(\boldsymbol{m}^{(l)}\right)$, the third one that with the eight offsprings $\boldsymbol{m}^{+}\left(\boldsymbol{m}^{(l)}\right)$. For convenience, we introduced the scaled amplitudes $\hat{u}_{i}\left(\boldsymbol{p}^{(l)}, \boldsymbol{m}^{(l)}\right)=V_{i}^{-1 / 2} u_{i}\left(\boldsymbol{p}^{(l)}\right.$, $\left.\boldsymbol{m}^{(l)}\right)$ with the box volume $V_{l}=\left(2 \pi L \times 2^{-l}\right)^{3} . \hat{f}_{i}$ is the driving force, representing the boundary conditions, non-statistical, and specified later. The contributions of the $\boldsymbol{x}$-derivatives of the characteristic functions would generate both interactions between more distant levels as well as spatial interactions within a level; they are neglected in the present approximation.

The mode amplitude equations imply balance equations for the energy

$$
\hat{E}_{m}^{(l)}(t)=\frac{1}{2} \sum_{\boldsymbol{p}^{(l)} \in \mathrm{K} /}\left|\hat{\boldsymbol{u}}\left(\boldsymbol{p}^{(l)}, \boldsymbol{m}^{(l)}, t\right)\right|^{2}
$$

reading

$$
\begin{gathered}
\frac{\partial \hat{E}_{m}^{(l)}(t)}{\partial t}=\hat{T}_{\boldsymbol{m}}^{l-1 \rightarrow l}-\frac{1}{8} \sum_{\boldsymbol{m}^{+}} \hat{T}_{\boldsymbol{m}^{+}+(\boldsymbol{m}(l))}^{l+1} \\
-\nu \sum_{\boldsymbol{p}^{(l)}} \boldsymbol{p}^{(l) 2}\left|\hat{\boldsymbol{u}}\left(\boldsymbol{p}^{(l)}, \boldsymbol{m}^{(l)}\right)\right|^{2} .
\end{gathered}
$$

Here $\hat{T}_{m}^{l-1 \rightarrow l}$ is the energy transfer into level $l$, box $\boldsymbol{m}^{(l)}$. Summing $V_{l} \hat{E}_{\boldsymbol{m}}^{(l)}$ on $\boldsymbol{m}^{(l)}$ and $l$ gives the energy carried by the field (2), which for $\nu=\hat{f}=0$ is conserved.

We will call eqs. (4) the "multifractal" approximation to the Navier-Stokes equation since it allows for different types of scaling in different regions in space. Correspondingly, our previous [12] approximation with spatial coherence in the periodicity box (but temporal chaos) will be termed "monofractal" turbulence.

To completely define the turbulent state we specify the deterministic force

$$
\begin{aligned}
& \hat{f}_{i}\left(\boldsymbol{p}^{(l)}, \boldsymbol{m}^{(l)}\right) \\
& \quad=\frac{1}{12} \epsilon \sum_{\boldsymbol{p}^{(0)} \in \mathrm{K}_{\mathrm{in}}} \delta_{\boldsymbol{m}(l), \boldsymbol{m}(0)} \delta_{\boldsymbol{p}(l), p^{(0)}} \frac{\hat{u}_{i}(\boldsymbol{p}, \boldsymbol{m})}{|\hat{\boldsymbol{u}}|^{2}} .
\end{aligned}
$$

$K_{\text {in }}$ is [12] a six-mode subset of $K_{0}$. The forcing happens on the largest scale only and provides constant energy input rate $\epsilon$.

Due to the spatial branching the number of mode amplitudes in (4) increases exponentially with the range of scales, i.e. with the number of levels $l$. To keep the set of o.d.e.'s tractable for a sufficiently extended inertial subrange (high $\mathrm{Re}$ ) we introduce now pruning. First, put $\nu=0$ (to simulate pure inertial range) and drain energy on the smallest scale level $l=N_{\eta}$ only, by introducing into (4) an eddy viscosity (in analogy to ref. [15])

$$
\begin{aligned}
& \nu_{\text {eddy, } i}^{(l)}\left(\boldsymbol{p}, \boldsymbol{m}^{(l)}\right) \\
& \quad=-D \times 2^{l}\left|\hat{\boldsymbol{u}}\left(\boldsymbol{p}_{1}^{(l)}, \boldsymbol{m}^{(l)}\right)\right| \hat{u}_{i}\left(\boldsymbol{p}_{2}^{(l)}, \boldsymbol{m}^{(l)}\right) \delta_{\boldsymbol{p}, \boldsymbol{p}_{2}^{(l)}},
\end{aligned}
$$

which is negative definite in the balance equation (5) and of the same dimension as the transfer terms. $\boldsymbol{p}_{1}^{(l)}$ refers to $2^{l}(-1,2,2), \boldsymbol{p}_{2}^{(l)}$ to $2^{l}(2,2,2) . D$ will be chosen self-consistently. Eqs. (4) represent a tree structure, cf. fig. 1a, so far. Secondly we now prune the tree, see fig. $1 \mathrm{~b}$. The number of boxes on
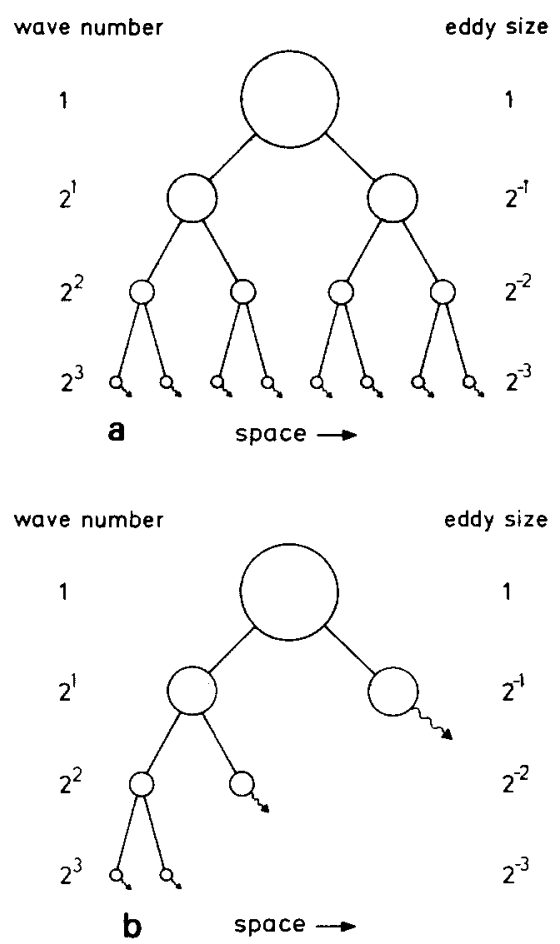

Fig. 1. (a) The coupling structure of the o.d.e. set for the NavierStokes equation. We indicated only two of the eight offsprings every box has in three dimensions. The $/$ th level has $2^{37}$ boxes (represented by bubbles) each consisting of 52 real mode amplitudes. The interactions are represented by lines, the wavy lines denoting draining by eddy viscosity. (b) The "pruned" version of (a). From all but one spatial box the energy is drained by the eddy viscosity (7), $\propto D$. Only one box is branching again, etc. 
each level $l$ now again is constant (namely, 8 , for all $l>0$ ), while the full series of branchings is still present in an increasingly smaller portion of real space. The eddy viscosity strength $D$ is uniquely determined by adjusting the average energy transfer to be the same through all branches on each level. Energy draining by eddy viscosity then equals the "natural" draining by the interlevel coupling. From our simulations we determined $D=0.78$.

\section{Spatial spottiness}

Solutions of the "monofractal" set of mode amplitude equations are exact solutions (with $\hat{\mathfrak{u}}_{i}\left(\boldsymbol{p}^{(l)}\right.$, $\left.\boldsymbol{m}^{(l)}\right)$ independent of $\left.\boldsymbol{m}^{(l)}\right)$ of the "multifractal" equations of motion (4). We now study what happens if we slightly disturb a spatially homogeneous solution of (4). This we do in a two-level system, one box with $l=0$, eight boxes with $l=1$, draining the second level by the eddy viscosity. The initial value of one amplitude out of the 52 real mode amplitudes in one box is disturbed by $0.1 \%$. The time evolution of the other seven boxes is kept identical, by symmetry. Fig. 2 shows how the difference between the energy of the initially perturbed box to that of another box evolves in time. After about three large eddy turnover times the initially minute disturbance has reached macroscopic size, the typical behaviour of trajectory divergence in nonlinear dynamical systems. The corresponding Lyapunov exponent ap-

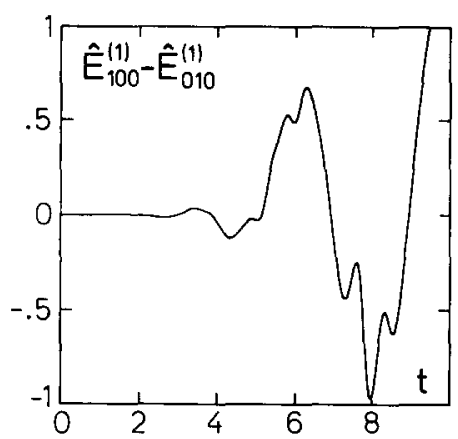

Fig. 2. The time evolution of a subbox subject to a small initial perturbation. Drawn is the difference in energy between the perturbed box $m^{(1)}=(1,0,0)$ and one of its seven identical siblings, $\boldsymbol{m}^{(1)}=(0,1,0)$. The time units are (and henceforth will be) turnover times $\left(L^{2} / \epsilon\right)^{1 / 3}$ of the largest eddy. parently is of the order of (turnover time) ${ }^{-1}$.

Next we look at the independent, non-constrained evolution in all eight real space boxes over a longer stretch of time, fig. 3. It is evident that the probability for large excursions from the mean value has increased in the individual boxes, while the average $E^{(1)}$ over the eight boxes, i.e. over the complete periodicity volume, resembles the top level, $E^{(0)}$ (scaled in magnitude and time). $E^{(1)}$ resembles the monofractal case, where the absence of branching enforces an average in space.

Let us address now the question if the fluctuations will tend to amplify when the branching is repeated. The answer comes from numerical simulation of the pruned (cf. fig. 1b) six-level "multifractal" cascade. The value of

$$
\begin{aligned}
& \Delta u_{i}^{(l)}(t)=\sum_{\boldsymbol{p}^{(l)} \in \mathbf{K}_{l}}\left[\operatorname{Re}\left\{\hat{u}_{i}\left(\boldsymbol{p}^{(l)}, \boldsymbol{m}^{(l)}\right)\right\}\right. \\
& \quad \times\left[\cos \left(p_{i}^{(l)} \times 2^{-l}\right)-1\right] \\
& \left.+\operatorname{Im}\left\{\hat{u}_{i}\left(\boldsymbol{p}^{(l)}, \boldsymbol{m}^{(l)}\right)\right\} \sin \left(p_{i}^{(l)} \times 2^{-l}\right)\right]
\end{aligned}
$$

was recorded for all components $i$ and each box $\boldsymbol{m}^{(l)}$ of level $l$, i.e. for each eddy of size $\approx 1 / p^{(l)}$. Its physical meaning also is the saddle point approximation with respect to $l$ of the longitudinal velocity difference $u_{i}\left(2^{-l} e_{i}\right)-u_{i}(0)$. The time evolution of $\Delta u_{i}^{(l)}(t)$ provides the probability distribution (pdf) for the velocity differences coming from level $l$ in any box $\boldsymbol{m}^{(l)}$, i.e. on scale $2^{-!}$.

As fig. 4 shows, the largest eddy level $l=0$ has an

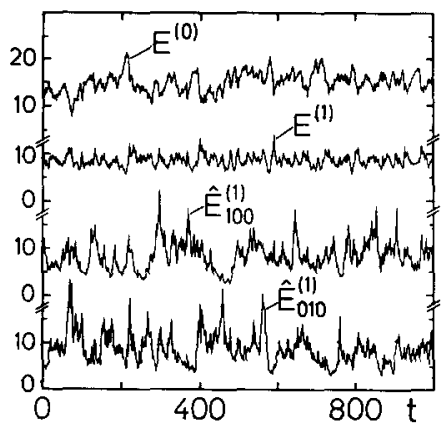

Fig. 3. The time evolution of a two-level system. Shown is from top to bottom: the energy of the highest level (one box), fed by constant energy input; the average energy of the eight subboxes (level 1); the individual energies of two of the subboxes. The

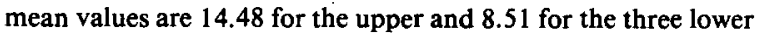
signals. 


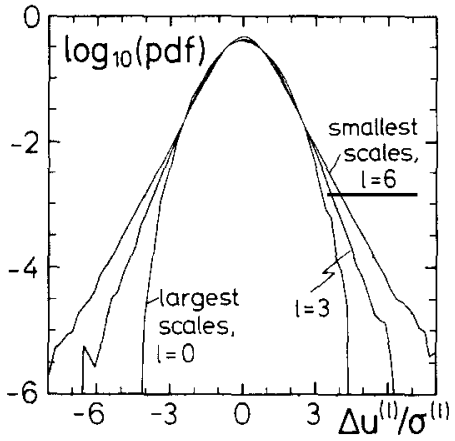

Fig. 4. The probability distributions of the velocity difference ( 8 ) for three different scales $2^{-l}, l=0,3,6$. The data are taken from a simulation of the six-level system over 16000 turnover times. All pdf's are normalized and scaled to unit variance.

almost Gaussian pdf, while the smaller eddies' pdf's develop increasingly stretched tails. These exponential-type tails are one of the trademarks of experimental identification of intermittency $[3,18]$.

We computed the moments of $\Delta u_{i}^{(l)}$ (time averaging). As an exact consequence of Kolmogorov's structure equation it is $D^{(3)}(r) \propto r$, thus $\left.\log _{2}\left(\left\langle\left|\Delta u_{i}^{(\prime)}\right|^{3}\right\rangle\right\rangle\right)$ should scale like $l$. Numerically, this is not exactly satisfied. We therefore rescaled $\Delta u_{i}^{(l)}$ once for every scale $l$ as to reproduce the correct scaling of the third moments $(\propto l)$. The other moments of order $m$ yield the $\zeta(m)$. Their values are summarized in table 1. Comparison with the data [2] is quite satisfactory.

We finally report on the numerical value of $b$ in the second order structure function $D^{(2)}(r)=$ $b(\epsilon r)^{2 / 3}(r / L)^{\delta \zeta(2)}$. In the "monofractal" case [12] with 52 real amplitudes per level we had $\delta \zeta(2) \approx 0$ and $b \approx 300$. The branching of the "multifractal" equations enhances the energy transport leading to $b \approx 150$ (experimentally $b_{\exp }=8.4$ ). While the scal- ing exponents are already convincing, $b$ is not yet.

\section{Discussion}

In view of the equations of motion (4) for the mode amplitudes of the Navier-Stokes velocity field the following picture of the mechanism for intermittency arises. The energy of an $l$-level eddy has to be shared by eight offsprings. These are in competition. If by chance the energy transfer into one subbox is high, there is less energy for the others. Such privilege in energy tends to amplify since the transfer rate is bilinear (or "autocatalytic") in the amplitudes. Therefore privileged subeddies gain even more intensity, nonprivileged ones loose. The result is spottiness. Due to the specific nonlinear interactions the system does not tend to the survival of the fittest but shows a deterministic chaotic behaviour. Thus, simultaneously with creating spottiness of the active region the Navier-Stokes interaction (whose Galilean invariance, i.e. whose Lagrangian character of fluid flow, is kept [12]) creates the stochastics of the turbulent field. There is no artificial stochastics added by forcing with noise, no adjustable model parameter (as in previous intermittency models [6-9]) is free. Having selected the modes everything is Navier-Stokes determined.

We found in ref. [12] that the Navier-Stokes interaction seems to enforce classical scaling $\zeta_{\mathrm{cl}}=m / 3$. Since the same $p$-space interactions are kept in the "multifractal" analysis it is only the branching of the cascade that induces small deviations $\delta \zeta(\mathrm{m})$.

There are various refinements of our theory. For instance, with finite viscosity $\nu$ we expect $D$ to become a function of both $\nu$ and level $l, D=D(\nu, l)$.

Table 1

The structure function exponents $\zeta(m)$ for $m=2,4,6$ and 8 . A least squares fit was made to the first five levels of six levels simulated. Given is the statistical $\left(\chi^{2}-\right)$ error. The second line contains the experimental results of Anselmet et al. [2]. This is the first comparison between data and Navier-Stokes based theoretically computed $\zeta(m)$.

\begin{tabular}{lcccc}
\hline Exponent & $\zeta(2)$ & $\zeta(4)$ & $\zeta(6)$ & $\zeta(8)$ \\
\hline 6-level & 0.6982 & 1.2688 & 1.7028 & 1.9874 \\
calculation & \pm 0.0003 & \pm 0.001 & \pm 0.01 & \pm 0.04 \\
experiment [2] & 0.71 & 1.33 & 1.80 & 2.22 \\
\hline
\end{tabular}


The smallest size level $l=N_{\eta}$ should be chosen such that for given $\epsilon$ the amplitude $u_{i}^{\left(N_{\eta}\right)}$ is practically zero, hence $D\left(\nu, N_{\eta}\right)=0$. If $l \ll N_{\eta}, D$ should converge to its $\boldsymbol{\nu}$-independent fixed point, whose value is 0.78 for our 52 amplitudes-per-eddy mode selection.

We have made three types of approximations to the Navier-Stokes equation, which still have to be studied in greater detail: (i) Only interactions are included that are local in wavenumber space. (ii) The respective eight offsprings do not interact directly, but only indirectly via their common predecessor. One expects that adding direct spatial mixing will tend to reduce the fluctuations, cf. refs. [11,15]. (iii) Our eddy viscosity (7) only leads to energy loss, thus neglecting the backscattering effects [17] present in the complete transfer terms [12]. Hence the pruning approximation will tend to suppress fluctuations. The question remains open if this seriously affects the exponents.

\section{Acknowledgement}

One of us (JE) thanks the Deutsche Forschungsgemeinschaft for a postdoctoral fellowship and the Department of Mathematics of the University of Chicago for their hospitality. Mike Shelley supplied us with computer time. Finally, JE likes to thank Reuven Zeitak for numerous insightful discussions.

\section{References}

[1] A.N. Kolmogorov, C.R. Acad. Sci. URSS 30 (1941) 299; A.M. Obukhov, C.R. Acad. Sci. URSS 32 (1941) 19; C.F. von Weizsäcker, Z. Phys. 124 (1948) 614; W. Heisenberg, Z. Phys. 124 (1948) 628; L. Onsager, Phys. Rev. 68 (1945) 286.

[2] F. Anselmet, Y. Gagne, E.J. Hopfinger and R.A. Antonia, J. Fluid Mech. 140 (1984) 63.

[3] Y. Gagne, E.J. Hopfinger and U. Frisch, in: New trends in nonlinear dynamics and pattern forming phenomena, eds. P. Huerre and P. Coullet (Plenum, New York, 1988).

[4] R.H. Kraichnan, J. Fluid Mech. 5 (1959) 497; Phys. Fluids 8 (1965) 575.

[5] H. Effinger and S. Grossmann, Z. Phys. B 66 (1987) 289.

[6] A.N. Kolmogorov, J. Fluid Mech. 13 (1962) 82; A.M. Obukhov, J. Fluid Mech. 13 (1962) 77.

[7] B.B. Mandelbrot, J. Fluid Mech. 62 (1974) 331.

[8] U. Frisch, P.L. Sulem and M. Nelkin, J. Fluid Mech. 87 (1978) 719

[9] R. Benzi, G. Paladin, G. Parisi and A. Vulpiani, J. Phys. A 17 (1984) 3521.

[10] M.H. Jensen, G. Paladin and A. Vulpiani, Phys. Rev. A 43 (1991) 798.

[11] R.H. Kraichnan, J. Fluid Mech. 62 (1974) 305.

[12] J. Eggers and S. Grossmann, Does deterministic chaos imply intermittency in fully developed turbulence?, Phys. Fluids $A$, in press.

[13] J. Eggers and S. Grossmann, Phys. Lett. A 153 (1991) 12.

[14] S. Grossmann, Physica 29 (1963) 1373; 30 (1964) 779.

[15] E.D. Siggia, Phys. Rev. A 15 (1977) 1730; R.M. Kerr and E.D. Siggia, J. Stat. Phys. 19 (1978) 543.

[16] F.F. Argoul, A. Arnéodo, G. Grasseau, Y. Gagne, E.J. Hopfinger and U. Frisch, Nature 338 (1989) 51;

C. Meneveau, Analysis of turbulence in the orthogonal wavelet representation, CTR Manuscript 120 (1991).

[17] C. Meneveau, Phys. Rev. Lett. 66 (1991) 1450.

[18] B. Castaing, Y. Gagne and E.J. Hopfinger, Physica D 46 (1990) 177 